\title{
PESO AO NASCER E CRESCIMENTO FÍSICO DO LACTENTE
}

\author{
BIRTHWEIGHT AND INFANT GROWTH
}

Antonio A. Barros Filho', Marco A. Barbieri², Manoel R. P. Gutierrez ${ }^{3}$, Heloísa Bettiol', Rubens G. Ricos'

Docente' do Departamento de Pediatria - Faculdade de Ciências Médicas - UNICAMP, Docente ${ }^{2}$ e Médico Assistente ${ }^{3}$ do Depas to de Puericultura e Pediatria da Faculdade de Medicina de Ribeirão Preto da Universidade de Săo Paulo.

Corresponóncia: Heloísa Bettiol, Departamento de Puericultura e Pediatria da Faculdade de Medicina de Ribeirâo Preto da U dade de Săo Paulo - Campus Universitário - CEP: 14048-900 - Ribeirão Preto - SP;FAX: (016) 633-0136; E-mail: hbettiol @fmrp

BARROS FILHO AA. et al. Peso ao nascer e crescimento físico do lactente. Medicina, Ribeirão Preto. 29: 258-268, abr/set., 1996.

RESUMO: Esta revisão objetiva discutir a influência do peso ao nascer sobre o crescimento do lactente. Os autores analisam e discutem alguns fatores relacionados com o peso, ao nascer, ao crescimento, à recuperação do crescimento dos recém-nascidos de baixo peso, descritos na literatura internacional, e descrevem alguns estudos brasileiros. Chamam a atenção para alguns pontos controvertidos na literatura, bem como para o pequeno número de pesquisas desenvolvidos no Brasil.

UNITERMOS: Peso ao Nascer. Crescimento-Lactente. Peso-Estatura.

\section{INTRODUÇÃO}

A vigilância do crescimento - avaliação sistemática da evolução do peso e comprimento de crianças - uma estratégia simples e eficaz contida nos planos de assistência integral à saúde materno-infantil, em atenção primária à saúde, é recomendada pela Organização Mundial da Saúde, para auxiliar na identificação precoce de qualquer processo que incida sobre o crescimento ${ }^{1}$

O princípio dessa vigilância reside no fato de que os incrementos positivos de peso e comprimento, em um dado intervalo de tempo, determinam curvas ascendentes em função da idade (tempo). Esse perfil ascendente e suas variações refletem, em princípio, a complexa e contínua interação da criança (genética) com seu meio (social e ecológico) 2, $^{3}$. Herança, neste sentido, representa a capacidade geneticamente determinada da criança em adaptar-se e responder, inclusive metabólica e imunologicamente, às exposições, estímulos e solicitações do seu meio ambiente. por sua vez, é continente dos processos e das rel sociais, configuradas na sociedade na qual a c irá crescer e se desenvolver ${ }^{4,5}$. Aquele perfil peportanto, inferir o processo do crescimento, en presentado cartesianamente, com adequada apro ção e sensibilidade. No entanto, para uma com são satisfatória desse processo, durante a monito do crescimento, fundamentada nessas observaçố para estabelecer prognóstico sobre o crescime infância, é imprescindível compreender o cresc dos lactentes durante os dois primeiros anos de Esse período é caracterizado por rápidas transf ções e necessidades nutricionais específicas, o crescimento reflete mais a influência do "meios biente materno" (útero) do que as influências cas e do "meio ambiente externo"6, 7

As pesquisas e avanços tecnológicos ma médica, o acesso das gestantes à assistência mé educativa ${ }^{8.9}$ e o desenvolvimento de centros espe 
Endos para cuidados intensivos de recém-nascidos, Enalterado profundamente as expectativas de sobreanzincia das crianças pré-termo e, conseqüentemen= a morbidade e mortalidade desse grupo - até en- a principal causa de mortalidade infantil nos desenvolvidos. Isso coloca em pauta novas mestóes relativas ao crescimento e desenvolvimento ase grupo específico.

Neste trabalho, tomando como objeto o crescizento pós-natal de crianças pré-termo (desnutridas ou e, como ponto de partida, seu tamanho, ao nas- objetiva-se discutir esses aspectos, e o estado li do conhecimento nessa área, no Brasil.

\section{AVALIAÇÃO DO CRESCIMENTO INTRA-UTERINO E PESO AO NASCER}

Alguns estudos sobre recém-nascidos (RN) com peso $(<2500 \mathrm{~g})$, realizados nos anos $60 / 70$, mosznm que, após algum tempo de vida, para um grupo z crianças, o peso tendia a aproximar-se do peso de Ls rascidos com mais de $2500 \mathrm{~g}$, enquanto que isso =corria para outros. Essa observação levou TTAGLIA \& LUBCHENCO, $1967^{10}$, elegendo o $10^{\circ}$ -nili da curva de referência de LUBCHENCO, $1963^{11}$, anto ponto de corte, a proporem a denominação de ablequados para a idade gestacional" (AIG) para os Timeiros (com peso ao nascer entre os percentis 10 x. "pequenos para a idade gestacional" (PIG) para segundos (peso ao nascer abaixo do $10^{\circ}$ centil, Żermo ou não, mas com evidente desnutrição in-itero) e "grandes para a idade gestacional" (GIG) Tso acima do $90^{\circ}$ centil).

O uso de "curvas" e a escolha dos percentis de te, ou limítrofes, para avaliação do crescimento de encerram controvérsias ${ }^{12,13,14}$. Porém, a curva rposta por LUBCHENCO é a base dos modernos atroses de crescimento fetal ${ }^{15}$ - embora usada para Irbos os sexos e não levar em conta a paridade. Ou= curvas consideram o sexo do $\mathrm{RN}$, a paridade e a an das mães ${ }^{16}$, e alguns países ou mesmo serviços E aeonatalogia usam curvas próprias ${ }^{1720}$.

As crianças com baixo peso ao nascer (pré-ters ou não), porém, presumivelmente, com desnutriz intra-útero, podem ainda ser avaliadas por outros netsos, como por exemplo, pelo "índice ponderal =23hrer" $\left[\mathrm{IP}=\left[\right.\right.$ peso $\left.(\mathrm{g}) / \mathrm{comp}^{3}(\mathrm{~cm})\right] \times 100$ - que re$=0$ crescimento intra-uterino. Segundo este índiRN podem ser divididos em: 1 . simétricos - com índice dentro dos limites esperados, com peso proporcional ao comprimento, ou desnutridos crônicos; 2. assimétricos - com índice inferior ao limite esperado (com maior comprometimento do peso) ou desnutridos agudos ${ }^{21,22}$. Esse índice tem a vantagem de não necessitar da idade gestacional, que freqüentemente constitui uma importante fonte de erro. No entanto, alguns autores consideram que as crianças "simétricas" podem ter o tamanho determinado por fatores genéticos ${ }^{13}$. Para maior sensibilidade, o IP deve ser usado em associação com o peso de nascimento, e não tomado em relação a um valor standard de uma população normal, uma vez que crianças com peso aparentemente normal podem apresentar retardo do crescimento ${ }^{23}$. Entre a $30^{a}$ e $40^{\mathrm{a}}$ semanas de gestação, o percentil 50 (Pc50) desse índice aumenta de 2,33 para 2,62 e o percentil $3(\mathrm{Pc} 3)$, de 2,00 para $2,20^{24}$.

Outros índices permitem avaliar o crescimento fetal e estimar o peso ao nascer ${ }^{25}$. Escores de maturidade baseados na circunferência do braço (MAC) e craniana (HC), cujo uso clínico permite distinguir RN pré-termos de baixo peso e retardo do crescimento de $\mathrm{RN}$ a termo, com peso baixo ${ }^{26}$; ou modelos que utilizam as circunferências toráxica e/ou braquial ${ }^{27,28}$.

\section{CRESCIMENTO DOS RN DE BAIXO PESO}

A recuperação do peso e o crescimento pós-natal das crianças pré-termo dependem de alguns fatores: $\mathbf{1}$. do tempo de gestação; $\mathbf{2}$. da causa que determinou a prematuridade; 3 . da assistência à gestante durante a gravidez, e da possibilidade de diagnosticar e controlar aquela causa ${ }^{29} ; 4$. da condição socioeconômica da família e seu meio ambiente ${ }^{30}$.

BABSON, $1970^{31}$, classificando RN segundo peso de nascimento (PN) e idade gestacional (IG) em 1. "muito prematuros" (PN: 950-1300g e IG: 27-29 semanas); 2. "moderadamente prematuros" (PN: 1400-2000g e IG: 31-33 semanas); 3. "a termo" mas com tamanho severamente comprometido ( $\mathrm{PN}<2000 \mathrm{~g}$ e IG $>37$ semanas), e, comparando as respectivas curvas com as consideradas normais, observou que, quando corrigidas para a IG, mantiveram-se ascendentes e paralelas, porém inferiores às referências.

SOUZA, $1992^{32}$, também observou que o crescimento em peso e comprimento de RN pré-termo (AIG), até o $6^{\circ}$ mês pós-termo, esteve sempre abaixo dos relatados por BRANDT, $1985^{29}$, e das referências do NCHS. 
FITZHARDINGE \& STEVEN, $1972^{33}$, acompanhando $\mathrm{RN}$ a termo e com severo retardo do crescimento intra-uterino, e comparando longitudinalmente o peso, comprimento e perímetro cefálico com referências normais, observaram que, aos 4 anos, o peso médio e o comprimento estavam entre o $10^{\circ}$ e o $25^{\circ}$ percentil, e $35 \%$ mantiveram-se abaixo do $3{ }^{\circ}$ percentil, enquanto que apenas $8 \%$ se encontravam acima do $50^{\circ}$. Os irmãos que nasceram com peso dentro dos limites da normalidade, tomados como controle, tenderam a ser maiores - $3 \%$ deles estavam abaixo do 3 centil e $45 \%$ acima do $50 \%$. Entretanto, a velocidade de crescimento foi semelhante aos normais. A evolução do perímetro craniano também foi semelhante ao do comprimento por idade.

CRUISE, $1973^{34}$, em um estudo longitudinal prospectivo acompanhou, até os 3 anos de idade, $220 \mathrm{RN}$ de baixo peso de parto único, caucasianos e saudáveis, e comparou-os com $113 \mathrm{RN}$ a termo, de peso acima de $2500 \mathrm{~g}$. Com relação à IG foram subdivididos em: grupo 1- de 28 a 32 semanas; grupo 2- de 33 a 36 semanas, com peso apropriado para a IG; grupo 3- de 37 a 42 semanas de gestação, porém PIG. Estes, embora tivessem medidas maiores que dos demais, aos 12 meses, haviam sido ultrapassados pelos dois outros gru- pos, tanto em velocidade como em comprimento em distância, e, aos 2 e 3 anos, eram os menores. A velocidade de crescimento de cada grupo de estudo foi maicr durante os três primeiros meses de vida. A velocidade de crescimento das crianças consideradas AIG, de cadi: sexo, foi maior que a dos considerados PIG, particularmente da circunferência craniana - ao nascimento, acs 3 meses, e dos 3 aos 6 meses. As diferenças de velocidade de crescimento para todas as medidas diminuie a partir do $6^{2}$ mês, porém as crianças AIG mantivera vंelocidades maiores que as crianças PIG, até os 3 ancs de idade. Resultados semelhantes também foram ct. servados por outros autores ${ }^{35 / 38}$.

BARBIERI, $1975,1976^{(39.40)}$, em um estud: prospectivo, acompanhou 142 lactentes, nascidos con peso $<2300 \mathrm{~g}$, até os 2 anos de idade. Observou que az crianças apresentaram recuperação do crescimenta mas mantiveram-se abaixo dos valores médios pan crianças normais, do mesmo nível socioeconômic tomando como referência as curvas propostas par GONÇALVES, $1971^{41}$. Na Tabela I, são apresentad̆ as distribuições das porcentagens observadas nos roo pectivos intervalos dos percentis, aos 6, 12 e 24 me ses (os RN não foram diferenciados entre prematura e desnutridos intra-útero).

Tabela I - Distribuição percentual dos RN de baixo peso, segundo os intervalos de percentis de peso e comprimento, idade e sexo (compilados de Barbieri, 1975)

\begin{tabular}{|c|c|c|c|c|c|c|}
\hline \multirow{3}{*}{ Idade } & \multirow{2}{*}{\multicolumn{6}{|c|}{ MASCULINOS }} \\
\hline & & & & & & \\
\hline & $\mathrm{Pc}^{*}<5$ & Pc 5-50 & $\mathrm{PC}>50$ & $\mathrm{Pc}<5$ & Pc5-50 & $P_{c}>50$ \\
\hline 6 & 59,5 & 30,9 & 9,5 & 58,0 & 39,0 & 3,0 \\
\hline 12 & 29,7 & 56,8 & 13,5 & 33,2 & 58,6 & 8,2 \\
\hline 24 & 29,1 & 54,2 & 16.7 & 25,6 & 66,4 & 8,0 \\
\hline \multicolumn{7}{|c|}{$\cdot \mathrm{P} c=$ percentil } \\
\hline \multirow{2}{*}{ Idade } & \multicolumn{6}{|c|}{ FEMININOS } \\
\hline & $P c^{*}<5$ & Pc5-50 & Pc $>50$ & $P_{C}<5$ & Pc5-50 & $P c>50$ \\
\hline 6 & 59,1 & 31,8 & 9,1 & 56,8 & 36,3 & 6,9 \\
\hline 12 & 31,4 & 57,1 & 11,5 & 34,1 & 54,3 & 11,6 \\
\hline 24 & 27,2 & 54,5 & 18,3 & 21,6 & 64,8 & 13,6 \\
\hline
\end{tabular}

$\cdot P c=$ percentil 


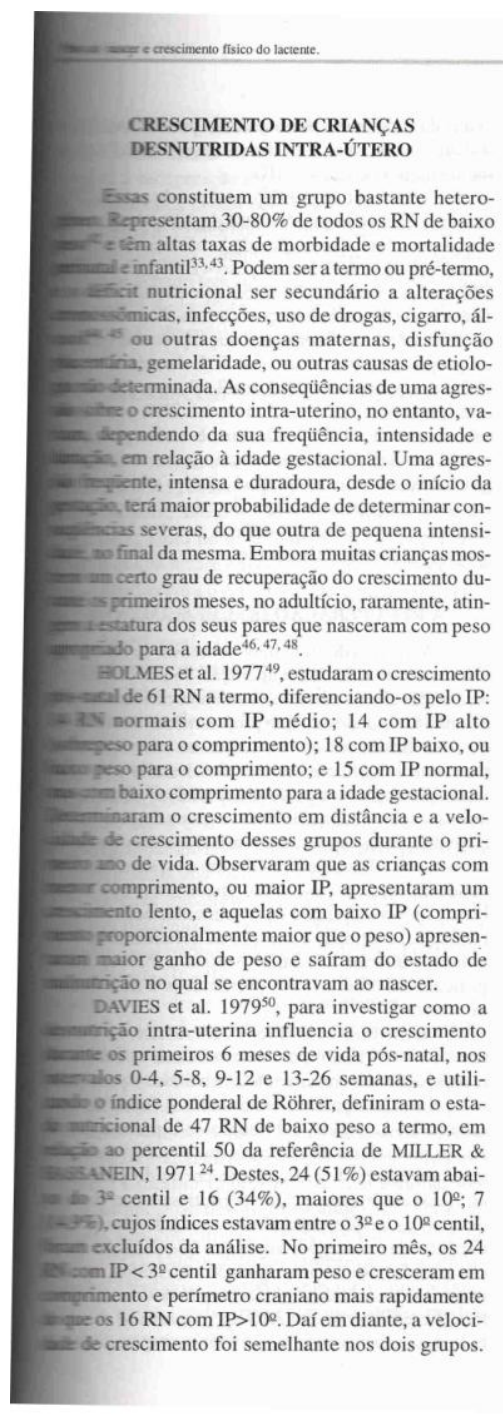

Comparando com um grupo de lactentes considerados normais ao nascimento, os autores observaram que, com exceção do peso no $1^{2}$ mês, para aqueles $\mathrm{RN}$ com IP $>10^{\circ}$ centil ao nascer, as taxas de crescimento (peso, comprimento e perímetro craniano) foram maiores que as dos normais, nos primeiros 3 meses, sugerindo que esses devem ser os meses de recuperação do crescimento dos lactentes com baixo peso ao nascer.

VILLAR et al., $1984^{51}$, estudaram o crescimento e desenvolvimento de 205 crianças, até os 3 anos de idade, divididas em dois grupos: 1- com peso normal ao nascer; 2- a termo, com desnutrição intra-uterina ou retardo do crescimento intra-uterino (IUGR). Este grupo foi subdividido em: 2.1- com índice ponderal baixo (IUGR-LPI); 2.2- com índice ponderal adequado (IUGR-API). As crianças destes subgrupos, ao nascerem, tinham pesos semelhantes, mas comprimentos e perímetros cranianos significativamente diferentes. Os lactentes IUGR-LPI apresentaram recuperação do peso, nos primeiros meses, às custas de depósito de gordura; os IUGR-API permaneceram mais leves e menores, e com o perímetro craniano menor até 30 meses. Aos 3 anos, os testes de desenvolvimento aplicados mostraram que as crianças com pesos normais apresentaram um pontuação mais alta que os IURG-API. Os autores concluíram que lactentes com baixo peso ao nascer tenderam a seguir um padrão de crescimento e desenvolvimento associado às suas características físicas ao nascimento.

FITZHARDINGE \& INWOOD, $1989^{46}$, estudaram o padrão de crescimento de 158 lactentes com retardo do crescimento intra-uterino durante os 2 primeiros anos de vida ( 84 com IG $>36$ semanas, dos quais 78 foram acompanhados e 74 com IG $<37$ semanas, dos quais foi possível o acompanhamento de 49 dos 61 sobreviventes - a taxa de mortalidade deste grupo foi de 18\%). Entre os primeiros, a aceleração do crescimento em peso começou logo após o nascimento e continuou por 6 meses (em média); o início da aceleração do crescimento linear foi mais tardia e foi limitada aos primeiros 9 meses; 23 lactentes (29\%) ainda estavam abaixo do $5^{\varrho}$ percentil, tanto de peso como de comprimento, aos 2 anos de idade. Entre os segundos, de IG<37 semanas, o peso de nascimento foi atingido por volta do $11^{\circ}$ dia, e a maior velocidade de ganho de peso ocorreu entre 4-6 semanas depois da data provável do parto, ou na que seria a data do termo da gestação. A recuperação do crescimento durou até 9 meses do termo; no entanto, aos 18 meses, $44 \%$ desses lactentes pré-termo estavam abaixo do $5^{\circ}$ percentil de peso, e $42 \%$ abaixo do $5^{2}$ percentil de comprimento. O tamanho, aos 18 meses pós-termo, 
com a idade corrigida, correlacionou-se com o peso à data do termo, e com o comprimento aos 3 meses, mas não com o grau de desnutrição intra-útero ou com o índice ponderal.

\section{CRESCIMENTO DE RN COM MUITO BAIXO PESO}

RN muito prematuros - IG $<30$ semanas - ou de muito baixo peso - $\mathrm{PN}<1500 \mathrm{~g}$ - terăo tamanho menor que crianças nascidas com peso normal ${ }^{52}$, embora também dentro deste grupo, crianças maiores terão tamanho maior $^{53}$.

HACK et al., $1984^{54}$, estudaram o crescimento e a recuperação de $182 \mathrm{RN}$ de muito baixo peso até a idade corrigida de 33 meses pós-termo. Ao nascimento, $147 / 182(80,8 \%)$ tinham o peso apropriado para a IG (peso $=$ média $\pm 2 D P$ ) e $35 / 182(19,2 \%)$, eram pequenos [peso < (média - 2DP)]. Esse mesmo critério foi utilizado para categorizá-los nas idades corrigidas de 40 semanas, 5, 21, e 33 meses. Dos 147 RN apropriados, $46 \%(67 / 147)$ corrigidos para 40 semanas, $27 \%(40 / 147)$ aos 8 meses, $19 \%(28 / 147)$ aos 21 , e $17 \%(25 / 147)$ aos 33 meses, apresentaram peso $<$ (média - 2DP) para a respectiva idade; o mesmo ocorreu com os 35 PIG, $91 \%$ (32/35) às 40 semanas, $49 \%$ $(17 / 35)$ aos 8 e 21 meses, e $46 \%$ (16/35) aos 33 meses. Os fatores correlacionados com o fracasso da recuperação do crescimento nos grupos dos AIG foram peso de nascimento, IG, gravidade das complicações neonatais e seqüelas físicas e neurológicas crônicas. Nos lactentes PIG, os fatores foram peso ao nascer, gemelaridade e classe social.

GILL et al. $1986^{55}$, investigaram o padrão de crescimento de $184 \mathrm{RN}$ sobreviventes, nascidos entre 23 e 29 semanas de gestação e parto único. Observaram uma perda de $14 \%$ do peso de nascimento em torno do $6^{\circ}$ dia de vida. Esta perda foi maior, bem como o tempo de recuperação, quanto maior a imaturidade - apesar do alto volume ingerido na primeira semana. Como resultado desta perda, o peso corporal médio, corrigido pela idade, manteve-se abaixo do $10^{\circ}$ centil da curva de crescimento intra-uterino. Da quarta semana pós-natal em diante, todos os subgrupos gestacionais apresentaram um ganho ponderal maior do que o referido como taxa de crescimento intra-uterino. Aos 2 anos de idade (corrigida para a prematuridade), e em uma amostra de 94\% (174/184) dessas crianças, não foram observadas diferenças de peso entre os classificados como AIG, mesmo quando de diferentes grupos de IG. O peso médio para os lactentes AIG e PIG estava comprendido entre o $3^{\circ}$ e o $10^{\circ}$ centil de uma curva de referência para crianças tralianas, embora os PIG tivessem peso significatit mente menor do que os AIG.

PIERETI et al., $1991^{56}$, analisando prospecti mente o crescimento pós-natal de $230 \mathrm{RN}$ de m baixo peso, até 3 anos de idade, divididos em di grupos - 1-com $60 \mathrm{RN}$ com peso $<1001 \mathrm{~g}$; 2 - com I RN com peso entre 1001 e $1500 \mathrm{~g}$, e usando como ferência as curvas de crianças normais do mesmo me socioeconômico, observaram que o peso das crian do grupo 1 estavam abaixo de $2 \mathrm{DP}$ da média, no meiro ano, e entre 1 e $2 \mathrm{DP}$, aos 2 e 3 anos. O peso crianças do grupo 2 estava entre 1 e 2DP, aos 12 ses, abaixo de 1DP, aos 2 anos, e muito próxin média normal, aos 3 anos. O comprimento não gou à média normal no grupo 1 , porém esta foi da, aos 2 anos, pelas crianças do grupo 2 . O perín cefálico estava na média normal, aos 3 e 2 anos, grupos 1 e 2 , respectivamente.

\section{A RECUPERAÇÃO DO CRESCIMENTO}

A maioria dos trabalhos sobre $\mathrm{RN}$ de baixo refere a existência da recuperação do seu cresci to, embora alguns autores considerem-na mais um de análise de dados do que propriamente um fení no - ao menos no que se refere ao crescimentio prematuros ${ }^{57}$. No entanto, ela foi descrita para rentes patologias e em diferentes idades ${ }^{58}$. Estud animais submetidos a diferentes graus de desnu e em diferentes idades, demonstraram sua ocort de maneira diferenciada conforme a espécie ${ }^{59}$, de dendo da idade do indivíduo, quando ocorreu o blema que constrangeu o crescimento, bem co etiologia, severidade e duração da deficiência do cimento anterior à terapia. Alguns trabalhos plificam o fenômeno em prematuros e desnutran intra-útero ${ }^{13,}$ 15, 54, 60.

KARNISKI et al. $1987^{57}$, estudaram 61 turos até os 29 meses para determinar se o concét recuperação do crescimento era válido - para comprimento e perímetro craniano. A idade de criança foi corrigida, subtraindo-se o número de manas prematuras da idade pós-natal, e foi usada criar um índice de crescimento corrigido para as variáveis (z-escore), que permitisse a comp de crianças de diferentes idades e sexo. Dados de cimento sem correções sugeriam que essa rec ção ocorria. No entanto, quando corrigidos, mavam-se dos obtidos do crescimento de crianças as mo. O crescimento dos AIG aproximava-se do rado para crianças normais a termo, enquanto 
75, aos 29 meses, ainda apresentavam um crescimenE subnormal, apesar da correção. Os autores concluí= que a recuperação do crescimento parece ser um Ejo estatística criada pela interpretação gráfica, ao s war a idade cronológica ao invés da idade apropriamente corrigida - tanto para os AIG, como para os 3a. Esse achado tem implicações importantes para o syimento de lactentes prematuros e pode afetar a terpretação diagnóstica de déficit de crescimento.

\section{O CRESCIMENTO DE LACTENTES}

A maioria dos estudos sobre a influência dos ifferentes fatores e suas interrrelações sobre o crescinento parte do pressuposto que a nutrição o afeta, e, zas maneira, são utilizados para avaliar o estado arricional, tanto no nível individual como coletivo. Tnhalhos que levam em conta o peso ao nascer estão nais voltados para o estudo do crescimento durante o 7eriodo pós-natal imediato $35,47,61$.

PAINE et al., $1983^{62}$, procuraram investigar os fifitos do peso ao nascer e da idade gestacional sobre zucrescimento. Acompanharam 262 crianças e avalia$\mathrm{am}$ os efeitos dessas duas variáveis independentes sib̆re o peso, comprimento e perímetro craniano, aos 4.8, 12, e 18 meses. Ambas as variáveis tiveram efeito a grificante sobre o peso, comprimento e perímetro Ianiano, aos 4 e 12 meses, enquanto que o crescinento intra-uterino teve efeito significativo para o peso tcomprimento, aos 18 meses de idade.

Alguns trabalhos descrevem o crescimento de sctentes brasileiros, correlacionando-o com as confiçōes socioeconômicas e com o aleitamento materso. As Tabelas II e III apresentam os dados de crescimento - comprimento e peso por idade e sexo - de betentes de 0 a 2 anos de idade, obtidos desses estub́s $^{63,64,65}$, comparados com os do NCHS ${ }^{66}$. Obserrando-se os dados de MARQUES(c) (de Santo André) e do NCHS(d), nota-se que as crianças brasileiras de bom nível socioeconômico apresentam comprimento epeso/idade praticamente idênticos aos das norte-amencanas ${ }^{67}$ e mesmo valores maiores em algumas idades (a justaposição desses dois estudos, de épocas diferentes, resultou em uma elevação da altura no estubo de 1982). Outros estudos também mostraram resultados idênticos, e estudos que incluíram populaçōes de baixa renda apresentaram valores médios menores que os relatados ${ }^{30,41,68}$.
BICUDO-ZEFERINO, $1992^{69}$, calculou os percentis 10,50 e 90 de comprimento e peso por idade e sexo, de dois grupos de crianças de 0 a 2 anos de idade, da cidade de Campinas (SP); 254 eram provenientes de um Centro de Saúde situado em um bairro periférico, e 229, de uma clínica privada. Comparando as curvas obtidas com as do NCHS, observou que as curvas das crianças procedentes da clínica privada sobrepunham-se às da referência, e as curvas das crianças do Centro de Saúde, desde o primeiro semestre de vida, apresentavam um afastamento tanto em relação às da clínica privada quanto às do NCHS. Aos 2 anos de idade, as diferenças entre os percentis 50 do comprimento era de $3,7 \mathrm{~cm}$ para o sexo feminino e $3,0 \mathrm{~cm}$ para o sexo masculino.

Estudos de MURAHOVSCHI et al., $1987^{70}$, sobre a influência do aleitamento materno sobre o crescimento de peso e comprimento de lactentes da cidade de Santos, SP, demonstraram que as médias das crianças, durante o aleitamento, eram menores que as referidas por MARQUES et al. ${ }^{64}$ e maiores que as do NCHS; BARROS, $1981^{71}$, em um município agroindustrial, observou que, em relação ao peso, as crianças sobrepunham-se às do NCHS, mas eram menores em comprimento. Discriminando os grupos de lactentes conforme a duração do aleitamento materno, observou ainda que este influenciava o peso, porém não interferia no comprimento.

Em relação ao aleitamento materno exclusivo e o crescimento de RN pré-termos, RAMASETHU et al., $1993^{72}$, analisaram, prospectivamente, o ganho de peso de 355 crianças de muito baixo peso durante as primeiras 24 semanas de vida, amamentadas por sua própria mãe, em uma enfermaria de cuidados especiais. Os pré-termos com peso entre 1000 e $1500 \mathrm{~g}$ dobraram seu peso de nascimento por volta da $10^{\mathrm{a}} \mathrm{se}-$ mana de vida e o triplicaram à $18^{\mathrm{a}}$, com velocidade de 20-30 g/dia até a $20^{\mathrm{a}}$; aqueles com peso de nascimento entre 1501 e $2000 \mathrm{~g}$, dobraram-no na $12^{\mathrm{a}}$ semana e o triplicaram na $16^{\mathrm{a}}$, com uma velocidade de $30 \mathrm{~g} /$ dia até a $11^{\mathrm{a}}$ e $20 \mathrm{~g} /$ dia após a $12^{\mathrm{a}}$. Os pré-termos nascidos com 32 semanas tiveram um período de atraso de cerca de 3 semanas, seguido por uma fase de rápido "catch up", enquanto que os nascidos entre 34 e 36 semanas tiveram um curto período de atraso de 2 semanas, seguido de uma fase de "catch up" semelhante aos anteriores. O peso atingido foi comparável à velocidade do crescimento intra-uterino. 
Tabela II - Distribuição de crianças, segundo o comprimento $(\mathrm{cm})$, sexo e idade (meses), em três estudos brasileiros e no NCHS.

\begin{tabular}{|c|c|c|c|c|c|c|}
\hline \multicolumn{7}{|c|}{ MASCULINOS } \\
\hline \multicolumn{3}{|c|}{ PNSN (a) em cm } & \multicolumn{2}{|c|}{ Marques (b) em cm } & \multirow{2}{*}{$\begin{array}{c}\text { Marques (c) em } \\
\mathrm{cm}\end{array}$} & \multirow{2}{*}{$\begin{array}{c}\mathrm{NCHS}(\mathrm{d}) \text { em } \\
\mathrm{cm}\end{array}$} \\
\hline Idade & Urbano & Rural & Classe I & Classe IV & & \\
\hline 0 & 51,5 & 50,4 & - & - & 50,1 & 50,5 \\
\hline 1 & 54,4 & 55,4 & - & - & $\cdot$ & 54,6 \\
\hline 3 & 62,8 & 61,1 & 60,0 & 61,0 & 62,7 & 61,1 \\
\hline 6 & 68,1 & 66,0 & 64,0 & 66,0 & 67,9 & 67,8 \\
\hline 9 & 70,7 & 69,5 & 68,0 & 71,0 & 72,6 & 72,3 \\
\hline 12 & 74,1 & 73,4 & 72,0 & 75,0 & 76,6 & 76,1 \\
\hline 18 & - & - & 78,0 & 82,0 & 83,4 & 82,4 \\
\hline 24 & 86,0 & 83,2 & 83,0 & 87,0 & 88,8 & 87,6 \\
\hline
\end{tabular}

\begin{tabular}{|c|c|c|c|c|c|c|}
\hline \multicolumn{7}{|c|}{ FEMININOS } \\
\hline \multicolumn{3}{|c|}{ PNSN (a) em cm } & \multicolumn{2}{|c|}{ Marques (b) em cm } & \multirow{2}{*}{$\begin{array}{c}\text { Marques (c) } \\
e \mathrm{~m} \mathrm{~cm}\end{array}$} & \multirow{2}{*}{$\begin{array}{l}\mathrm{NCHS} \text { (d) } \\
\mathrm{em} \mathrm{cm}\end{array}$} \\
\hline Idade & Urbano & Rural & Classe I & Classe IV & & \\
\hline 0 & 51,1 & 50,0 & $\cdot$ & - & 49,1 & 49,9 \\
\hline 1 & 54,9 & 53,9 & - & - & $\cdot$ & 53,5 \\
\hline 3 & 59,5 & 61,0 & 58,0 & 60,0 & 61,3 & 59,5 \\
\hline 6 & 65,5 & 64,8 & 63,0 & 65,0 & 66,4 & 65,9 \\
\hline 9 & 68,8 & 69,8 & 67,0 & 69,0 & 71,0 & 70,4 \\
\hline 12 & 74,6 & 71,5 & 71,0 & 73,0 & 75,1 & 74,3 \\
\hline 18 & - & - & 77,0 & 80,0 & 82,2 & 80,9 \\
\hline 24 & 83,5 & 81,9 & 82,0 & 86,0 & 88,0 & 86,5 \\
\hline
\end{tabular}

(a) INAN, 1990 (medianas)

(b) Marques et alli, 1974 (médias)

(c) Marques et alli, 1982 (medianas)

(d) Hamill (NCHS), 1977 (medianas) - OMS, 1983

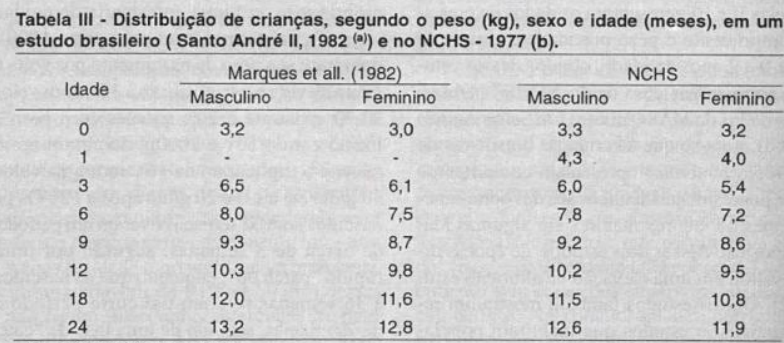

(a) - MARQUES et al., 1982

(b) - NCHS, 1977; OMS, 1983. 


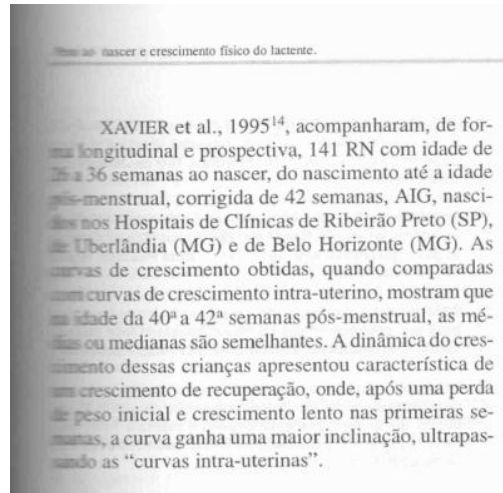

\section{CONSIDERAÇÕES FINAIS}

Em uma busca extensiva, na literatura, sobre ascimento e prognóstico dos RN de baixo peso ao acer, HOFVANDER, $1982^{73}$, encontrou que a grande = Eenvolvidos, e poucas de países em desenvolvitesto, onde a preocupação deveria ser maior, dada = maior incidência. Algumas pesquisas epidemioẩeas são expressivas. MONTEIRO, $1992^{74}$, obserque, entre as crianças brasileiras até 4 anos de tade, 10,2\% haviam nascido com menos do que $3 \times 0 \mathrm{~g}$, sendo $76,1 \%$ de termo e $23,9 \%$ prematuras, e a maioria desnutrida intra-útero. BETTIOL, $1995^{75}$, erificou que, na idade escolar, entre as crianças de arre coorte nascida de parto único hospitalar, em Ri=irio Preto, no período de um ano, foram encontraIs maiores proporçōes de crianças leves (peso/idade ahivo do Pc10 do NCHS), baixas (altura/idade abai$=$ do Pc10 do NCHS) e magras (peso/altura abaixo a Pe10 do NCHS) entre aquelas nascidas de baixo Per, as crianças da coorte consideradas pequenas ao Escer (com peso e comprimento ao nascer, simultaamente, abaixo de Pc10 do NCHS) tiveram maior probabilidade de ficar baixas, na idade escolar, do que as nascidas grandes (com peso e comprimento ao nascer, simultaneamente, maiores ou iguais ao Pc90 do NCHS), mostrando que as primeiras, provavelmente, sofreram efeitos adversos no seu crescimento intra-uterino e na vida pós-natal. Nesse estudo, envolvendo todos os nascimentos hospitalares de Ribeiräo Preto durante um ano, GOMES et al., $1990^{76}$, apontaram que $7,5 \%$ haviam nascido com peso inferior a $2500 \mathrm{~g}$, e $36,5 \%$ mediam até $49 \mathrm{~cm}$; em outro estudo, com os mesmos dados, GONÇALVES, $1992^{77}$, observou que, entre os RN menores que $2500 \mathrm{~g}, 47,4 \%$ eram pré-termo, e, entre os demais, $52,6 \%$ a termo, $75,8 \%$ eram PIG, e, entre estes, $56,9 \%$ eram simétricos e $43,1 \%$ assimétricos.

Em um estudo de coorte, VICTORA et al., $1988^{78}$, encontraram $9,0 \%$ de RN de baixo peso, sendo que $48,0 \%$ eram prematuros - percentual semelhante ao de Ribeirão Preto, porém maior que o observado por MONTEIRO, $1992^{74}$. No seguimento das crianças nascidas em 1982, e avaliadas aos 20 meses de idade, em relação às referências do NCHS, aqueles autores encontraram um maior comprometimento do crescimento entre as crianças com diagnóstico pregresso de PIG do que entre os pré-termos.

Embora vários centros de neonatalogia, no Brasil, tenham condições de atender RN menores que $1500 \mathrm{~g}$ dentro de padrōes de bom nível, não se tem relatado em que condiçōes essas crianças estão crescendo e se desenvolvendo. Esses estudos poderiam contribuir para uma melhor compreensão da dinâmica do crescimento em condições adversas, como também fornecer elementos para uma monitoração apropriada desse grupo populacional específico.

Alguns estudos mostram que algumas crianças de baixo peso exibem uma tendência de recuperação do crescimento nos primeiros meses de vida, enquanto que outras não. Seria por causa do potencial genético, ou consequeência de algum processo de desnutrição crônica intra-útero? Estas ainda são questões abertas.

BARROS FILHO AA et al. Birthweight and infant growth. Medicina, Ribeiráo Preto, 29: 258-268, apr/sept., 1996.

ABSTRACT: This review is aimed at discussing the influence of birthweight on subsequent growth of the infant. The authors discuss some factors related to birthweight, growth of the infant, growth of the infant. The authors discuss some factors related to birthweight, growth of the infariont, then they discribe some Brazilian researchs. They call the attention for some controvertial points found in the literature and for the lack of researchs on this issue in Brazil.

UNITERMS: Birth Weight. Infant Growth. Height Weight. 


\section{REFERÊNCIAS BIBLIOGRÁFICAS}

1 - ORGANIZACIÓN MUNDIAL DE LA SALUD. Conferencia de Alma-Ata sobre atención primaria de salud. Cronica de la OMS 32: 443-498, 1978.

2-MARSHAL WA. Human growth and its disorders. Academic Press, London, 1977.

3- MARTORELL R \& HABICHT JP. Growth in early childhood in developing countries. In: FALKNER F \& TANNER JM, eds. Human growth. A comprehensive treatise. 2th ed. Ple. num Press, New York, p.241-262, 1986.

4 - DANFORTH JrE. The role of thyroid hormones and insulin in the regulation of energy metabolism. Am J Clin Nutr 38 1006-1017, 1983.

5- ZEITLIN M. Nutritional resistence in a hostile environment: positive deviance in child nutrition. Nutr Rev 49: 259-268, 1991.

6. SMITH DW et al. Shifting linear growth during infancy: llustration of genetic factors in growth from fetal life through llustration of genetic factors in growth tro
infancy. J Pediatr 89: 225-230, 1976.

7. TANNER JM. Variability of growth and maturity in newborn infants. In: LEWIS M \& ROSEMBLUM L, eds. The effect of the infant on its caregiver. John Wiley \& Sons, London, p. $77 \cdot 103,1974$.

8- BLONDEL B et al. Poor antenatal care and pregnancy outcome. Eur J Obstet Gynecol Reprod Biol 50: 191-196. 1993.

9- HUESTON WJ. Prenatal care and low-birth-weight rates in urban and rural Wisconsin. J Am Board Farm Pract 8; $17-21,1995$

10 - BATTAGLIA FC \& LUBCHENCO LO, A practical classification of newborn infants by weight and gestational age. J Pediatr 71: 159-163, 1967.

11 - LUBCHENCO LO et al. Intrauterine growth as estimated from live bom birth-weight data at 24 to 42 weeks of gestation. Pediatrics 32: 793-800, 1963.

12 - TANNER JM. Use and abuse of growth standards. In: FALKNER F \& TANNER, JM, eds. Human growth. A comprehensive treatise. 2th ed. Plenum Press, New York, p.95-109, 1986

13 - DAVIES DP. Physical growth from fetus to early childhood. In: DAVIS J \& DOBBING J, eds. Scientific foundations of pediatrics. 2th ed. Heinemann, London, p.303-330, 1981.

14- XAVIER CC et al. Crescimento de recém-nascidos pré-termo. J Pediatr, Rio de Janeiro, 71:22-27, 1995.

15 - ALTIGANI M et al. Catch up growth in preterm intants. Acta Paediatr Scand, 357: 3-19, 1989. Suppl.

16 - TANNER JM \& THOMSON AM. Standards for birthweight at gestational periods from 32 to 42 weeks, allowing for maternal height and weight. Arch Dis Child 45: 566, 1970.
17 - BRENELLI MA \& MARTINS FILHO J. Curvas de crescime to intra-uterino da populaçăo de nascidos vivos na matedade do CAISM - UNICAMP J Pediatr, Rio de Janeiro, $=$ $21-25,1992$

18- NIKLASSON A et al. An update of the Swedish referent standards for weight, lenght and head circumference at birat given gestational age (1977-1981). Acta Paediatr Seart 80: 756-762, 1991.

19 - RAMOS JLA. Avaliação do crescimento intra-uterino medidas antropométricas do recém-nascido. Tese de D torado, Faculdade de Medicina da USP, Săo Paulo, 1 :

20-SALA MM. Estudo do crescimento intra-uterino na seat da metade da gestaçāo. Determinaçấo dos percentis 10.2 $50,75,90$, do peso placentário, peso e estatura fetal. de Livre Docência, Faculdade de Medicina de Ribeirăo Pr. to da USP, Ribeirăo Preto, 1977.

21- BELIZAN JM \& VILLAR J. EI crecimiento fetal y repercusion sobre el desarrollo del niño. In: CUMINSKr. MORENO EM \& SUAREZ OJEDA EN, eds. Crecimient: desarrollo. Hechos y tendencias. Organizac Panamericana de la Salud, Washington, p. 102-119, 1 ? (Publicación Cientifica, 510)

22 - LUBCHENCO LO; HANSMAN C \& BOYD E. Intraula growth in lenght and head circunference as estimated fislive births at gestational age from 26 to 42 weeks. Pesit rics 32: 403-408, 1966

23- CHARD T: COSTELOE K \& LEAF A. Evidence of gros retardation in neonates of apparently normal weigth. $J$ Oberet Gynaecol Reprod Biol 45: 59-62, 1992.

24 - MILLER HC \& HASSANEIN K. Diagnosis of impaires tu growth in newborn infants. Pediatrics 48: $511-522$, 13 ?

25 - DUDLEY NJ. Selection of appropriate uitrasound 5 for the stimation of fetal weight. Br J Radiol 68: 3es. 1995.

26- EREGIE CO. Determination of maturity at birth: further servation on a maturity scoring system for head circt ence and mid-arm circumference, East Afr Med 2 $48.50,1993$.

27 - HUQUE F \& HUSSAIN AM. Detection of low birthnewborn babies by anthropometric measuremernBangladesh. Indian J Pediatr 58: 223-231, 1991.

28 - WORLD HEALTH ORGANIZATION. Use of a sir anthropometric measurement to predict birth weight wit-test collaborative study of birth weight surrogates. Bull $\mathrm{x}^{\mathrm{n}}=$ Health Organ 71: 157-163, 1993.

29 - BRANDT I. Growth dynamics of low-birth-weight inthat Acta Paediatr Scand 319: 38-47, 1985. Supp

30 - JIMENES R; BERDASCO A \& MESA D. Evaluacion a influencia de factores socioeconomicos, maternos y a alimentacion, sobre el crecimiento de nin̂os con bajo znascer durante el primer año de vida. Rev Cubana fes 57: $412-427,1985$ 


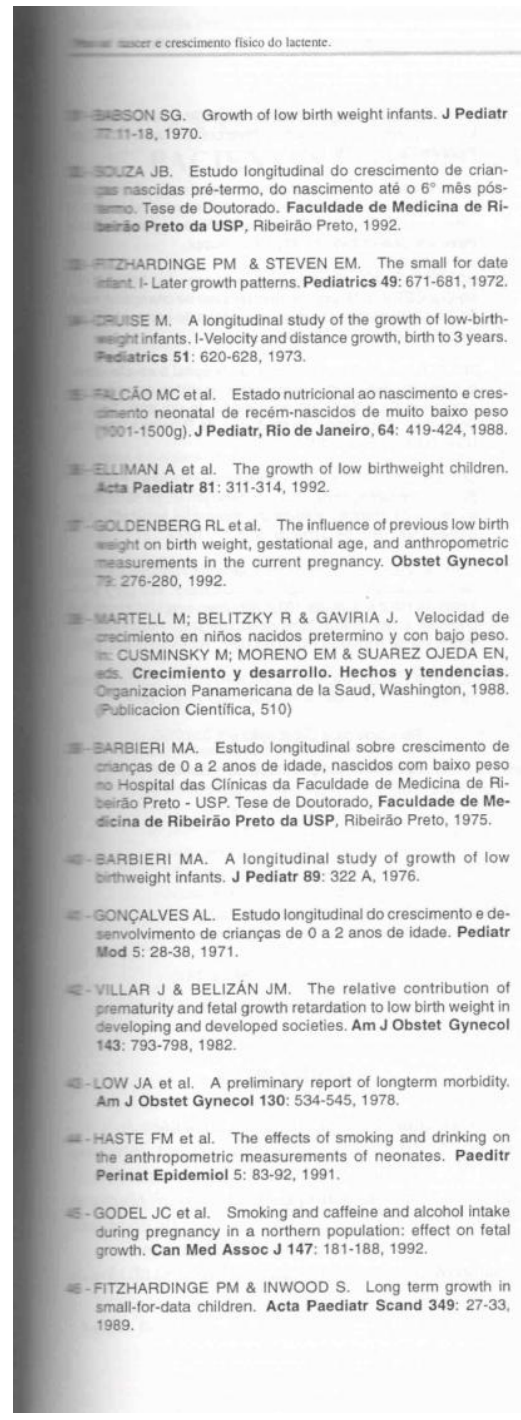

47 - WESTWOOD M et al. Growth and development of full-term nonasphyxiated small-for-gestational-age-selectd newborns follow-up through adolescence. Pediatrics 71: 376-382, 1983.

48. VILLAR J \& BELIZÁN JM. The timing factor in the pathophysiology of the intrauterine growth retardation syndrome. Obstet Gynec Surv 37: 499-506, 1982.

49- HOLMES GE et al. Postnatal somatic growth in infants with atypical fetal growth patterns. Am J Dis Child 131: 1078 $1083,1977$.

50- DAVIES DP et al. Nutritional status of light-for-date infants at birth and its influence on early postnatal growth. Arch Dis Child 54: 703-706, 1979.

51 - VILLAR $J$ et al. Heterogeneous growth and mental development of intrauterine growth-retarded infants during the first 3 years of life. Pediatrics 74: 783-791, 1984.

52- KIMBLE $J$ et al. Growth to age 3 years among very-lowbirth-weight sequellae-free survivors of modern neonatal intensive care. J Pediatr 100: 622-624, 1982.

53. KITCHEN WH: FORD GW \& DOYLE LW. Growth and the very low birth weight. Arch Dis Child 64: 379-382, 1989.

54- HACK $M$ et al, Catch-up growth in very-low-birth-weight infants. Am J Dis Child 138: 370-375, 1984.

55. GILL A et al, Postnatal growth in infants born before 30 weeks gestation. Arch Dis Child 61: 549-553, 1986.

56 - PIERETI MR et al. Postnatal growth of very-low-birth-weight newborn. Anthropomety after a period of 3 years - a longitudinal study. Rev Chil Pediatr 62: 285-289, 1991.

57-KARNISKI W: BLAIR C \& TUCCI JS. The illusion of catchup growth in premature infants. Am J Dis Child 141 520-526, 1987.

58- ORGANIZACIÓN MUNDIAL DE LA SALUD. Medición del cambio del estado nutricional. OMS, Genebra,1983.

58 - PRADER A; TANNER JM \& HARNACK GA. Catch-uP growth following illness or starvation. An exemple of devel. opmental canalization in man. J Pediatr 62: 646-659, 1963.

59- MCCANCE R A \& WIDDOWSON EM. The determinants of growth and form. Proc R Soc Lond 185: 1-17, 1974.

60- PIEKKALA P et al. The somatic growth of a regional birth cohort of 361 preterm infant during the first two years of life. J Perinat Med 17: 41.49, 1989.

61- JOAQUIM MCM et al. Crescimento ponderal de prematuros. J Pediatr, Rio de Janeiro, 52: 19-20, 1982.

62 - PAINE PA: PASQUALIL \& JOAQUIM MCM. Effects of birth weight and gestational age upon growth in Brazilian infants: a longitudinal study. J Trop Pediatr, 29: 11-17, 1983

63-MARQUES RM et al. Crescimento de crianças brasileiras. Peso $e$ altura segundo idade e sexo. Influência de fatores sócio-econômicos. Anais Nestlé, 1974. Suppl. 
64-MARQUES RM et al Crescimento e desenvolvimento pubertário em crianças e adolescentes brasileiros. II. Altura e peso. Editora Brasileira de Ciências, São Paulo, 1982.

65 - MINISTÉRIO DA SAÚDE. INSTITUTO DE ALIMENTAÇĀO E NUTRIÇÃO. Pesquisa Nacional sobre Saúde e Nutriçăo. Perfil de crescimento da populaçăo brasileira de 0 a 25 anos. Brasilia, 1990,

66- HAMILL PVV et al. NCHS growth curves for children birth18 years. Series 11, n.165. DHEW Publication. $N^{\circ}$ (PHS) $78-1650,1977$

67. SIQUEIRA AAF: ANDRADEJ \& ALMEIDA PAM. Peso, comprimento e perimetro cefálico de crianças brasileiras de classe social elevada. Rev Paul Med 97: 56-61, 1981.

68- MUSSO A \& MUSSO LK. O crescimento da criança no primeiro ano de vida no Estado da Guanabara. Bol Inst Univ Brasil 21: 161-180, 1964

69 - BICUDO-ZEFERINO AM. Fatores associados ao crescimento de crianças de 0 a 2 anos de idade no município de Campinas. Tese de Doutorado, Faculdade de Ciências Médicas da UNICAMP, Campinas, 1992.

70- MURAHOVSCHI $\mathrm{J}$ et al. Curvas e tabelas de crescimento de lactentes brasileiros de 0 a 6 meses de idade alimentados exclusivamente com leite materno. J Pediatr, Rio de Janeiro, 63: 1-23, 1987.

71 - BARROS FILHO AA. Crescimento, morbidade e leite materno. Estudo longitudinal no municipio de Pradópolis. Tese de Doutorado, Faculdade de Medicina de Ribeirăo Preto da USP, Pibeirấo Preto, 1981 .
72 - RAMASETHU J: JEYASEELAN L \& KIRUABAKARAN 0 Weight gain in exclusively breastfed preterm infants. $J \mathrm{Tr}=$ Pediatr 39: 152-159, 1993.

73- HOFVANDER,Y. International comparisons of postra growth of low-birth-weigth infants with special reference : differences between developing and affluent countries. $2=$ Paediatr Scand 296: 14-18, 1982. Suppl

74 - MONTEIRO MG. Baixo peso ao nascer. In: MONTE MFG \& CERVINI M, org. Perfil estatístico de crianças e - no Brasil. Aspectos de saúde e nutrição de crianças. Rio de Janeiro, p.11-18, 1992.

75 - BETTIOL H. Saúde da criança: do hospital à escola cimento de uma coorte de escolares nascidos de parto pitalar em Ribeirăo Preto no periodo de um ano. Teste $=$ Doutorado. Faculdade de Medicina de Ribeirâo Prete = USP, Ribeirăo Preto, 1995.

76- GOMES UA et al. Saúde perinatal em Ribeirăo Preto Brasil. Apresentaçăo de algumas caracteristicas demb́g sociais e da atenção médica da populaçẫo estudada. Saúde Públ, Rio de Janeiro, 6: 5-17, 1990 .

77 - GONCYALVES MB. Estudo da coorte de crianças nas: de baixo peso nos hospitais de Ribeirăo Preto (SP) junho de 1978 e maio de 1979. Algumas caracteristicas crianças $\theta$ das mäes. Tese de Doutorado. Faculdase Medicina de Ribeirão Preto da USP, Ribeirão Preto, 1 z

78 - VICTORA CG; BARROS FC \& VAUGHAN JP. Epidert da desigualdade. Editora Hucitec, Sāo Paulo, 1988.

Recebido para publicaçāo em 03/07/96

Aprovado para publicação em 22/08/96 